Classification

Physics Abstracts

$68.65-61.10 \mathrm{~F}-61.55 \mathrm{~F}$

Short Note

\title{
Cross section transmission electron microscopy observations of ultrathin films of cobalt in multilayers
}

\author{
Najeh Mliki( $\left.{ }^{2,1}\right)$, Danièle Renard $\left({ }^{3}\right)$, Monique Galtier $\left({ }^{3}\right)$ and Geneviève Nihoul $\left({ }^{1}\right)$ \\ ( ${ }^{1}$ ) G.M.E.T., Groupe Matériaux, University of Toulon, BP 132, 83957 La Garde Cedex, France \\ $\left({ }^{2}\right)$ INRST, Bordj Cedria, BP 95, 2050 Hammam Lif, Tunisia \\ $\left({ }^{3}\right)$ I.O, University of Orsay, 91405 Orsay Cedex, France
}

(December 02, 1991; accepted March 03, 1992)

\begin{abstract}
Résumé. - Nous présentons ici des résultats obtenus par microscopie électronique par transmission de profil sur des films ultraminces de cobalt (épaisseur descendant jusqu'à $0.5 \mathrm{~nm}$ ) séparés par des couches d'or. L'observation de profil montre que nos couches sont continues même pour les épaisseurs les plus faibles, ce qui n'avait pu être prouvé jusqu'alors.
\end{abstract}

\begin{abstract}
We present results obtained by cross section transmission electron microscopy on ultrathin cobalt films, i.e. with a thickness going down to $0.5 \mathrm{~nm}$, separated by gold layers. Observation of the profile views shows that our films are continuous, even for the smallest thickness, a fact which had not been proven until now.
\end{abstract}

\section{Introduction.}

Ultrathin ferromagnetic films (thickness around $1 \mathrm{~nm}$ ) or multilayers present properties of considerable interest, both from a fundamental point of view and from their potential applications in magnetic recording.The $\mathrm{Au} / \mathrm{Co}$ system is one of the most studied system, because it presents some very interesting and unusual properties.Stress induced effects, du to the $\mathrm{Au} / \mathrm{Co}$ lattice mismatch, as well as continuity and roughness of the films can play an important role on the stability of domain configuration. Hence, it is important to know the structure and morphology of the ultrathin cobalt films, on a local scale.

In a first paper, [1],HREM (High Resolution Electron Microscopy), and NMR (Nuclear Magnetic Resonance) were used to show the influence of the crystallographic characteristics of cobalt films on the resonance spectrum. Then, in situ, we measured variations of the free surface roughness and of the cobalt lattice constant during the cobalt deposition: these measurements were made, respectively, by SRX (Soft X ray Reflectometry) and RHEED (Reflection High Energy Electron Diffraction) [2] but they unfortunately, only give average results and are performed on a 
free cobalt surface: they tell us that the coverage of the gold layer is quite good and that the cobalt lattice is distorted, but this does not give information on a cobalt layer sandwiched between two gold layers, where the misfit is bound to give distorsions on both cobalt surfaces.

To obtain more information on the continuity of ultrathin cobalt films when positioned in a multilayer, we have now prepared cross sections of some samples. We report, here,our first results obtained by TEM (Transmission Electron Microscopy) on a multilayer (5 cobalt films) and on a bilayer, the cobalt thickness ranging from $0.5 \mathrm{~nm}$ to $2 \mathrm{~nm}$.

$\mathrm{The} \mathrm{Au} / \mathrm{Co}$ multilayers are fabricated in the same way as the $\mathrm{Au} / \mathrm{Co} / \mathrm{Au}$ sandwiches and their first characterization involve the same $X$ ray technique. More detailed information will be found in [3] for instance. Let us just recall some charasteristics of the first gold buffer: it is deposited on a float glass substrate, then annealed, its thickness being around $25 \mathrm{~nm}$. It is a textured polycrystalline film. The (111) closed-packed planes of the face centered cubic structure are preferentially parallel to the surface film and the lateral size of the grains is about $100 \mathrm{~nm}$. As we observe very thin cobalt films, this first gold layer will be our reference: in cross sections, the interesting orientations will be the $<111>$ and $<211>$ orientations which are perpendicular to the $<111>$ types.

The technique for obtaining cross section samples is now well know for semiconductors substrates but it is much more difficult to apply for multilayers on glass substrates as these tend to break easily. In the present case, an additional problem is the rather weak adherence of gold on glass.

\section{Penta-layer.}

In this multilayer,the five cobalt films have the same thickness $(2 \mathrm{~nm})$ and are separated by four $3 \mathrm{~nm}$ thick gold films. These values are obtained from a calibrated quartz monitor. The gold substrate is $25 \mathrm{~nm}$ thick while the final gold cover is about $100 \mathrm{~nm}$ thick.

Figure 1a is a bright field image of a cross section, taken using a 2000 FX Jeol microscope operating at a $200 \mathrm{kV}$ voltage. Colbat is a lighter element than gold and, for the small thicknesses involved here appears brighter than gold on the micrograph. More than $100 \mathrm{~nm}$ of the multilayer can be seen; we can see that all the layers are continuous but thicknesses are not the same everywhere, even for the first cobalt film, where the thickness varies between 1.5 and $2 \mathrm{~nm}$. The roughness of the gold substrate is weak, which is in good agreement with the results of many other measurements (see [2] for instance). But the roughness increases as the layers pile up, and defects are enhanced (see arrow). One can also see a grain boundary which crosses through the multilayer: there is a difference of intensity between the two grains in the substrate, in the cobalt films and in the gold cover. However, one can see that the existence of this grain boundary does not perturbe the cobalt layers which remain regular through the boundary: this has often been observed in this pentalayer and shows that the polycrystalline nature of the gold substrate does not perturb the magnetic layers. In the gold cover, which is farther from the hole and thus thicker, the contrast between the grains is smaller. Figure $1 \mathrm{~b}$ shows an intensity profile drawn across the pentalayer along with the abscissae for the maxima: one can see that the cobalt layers are rather regularly spaced and that their widths are very similar.

\section{Bilayer.}

In this bilayer, the first deposited cobalt film is very thin $(0.5 \mathrm{~nm}$, i.e. 2 atomic planes $)$ while the second evaporated film has a thickness of $1 \mathrm{~nm}$. They are separated by a gold layer of $5 \mathrm{~nm}$, while the gold substrate and the gold cover have the same thicknesses of $26 \mathrm{~nm}$.

Three bright field images of the cross section corresponding to the same sample are shown 


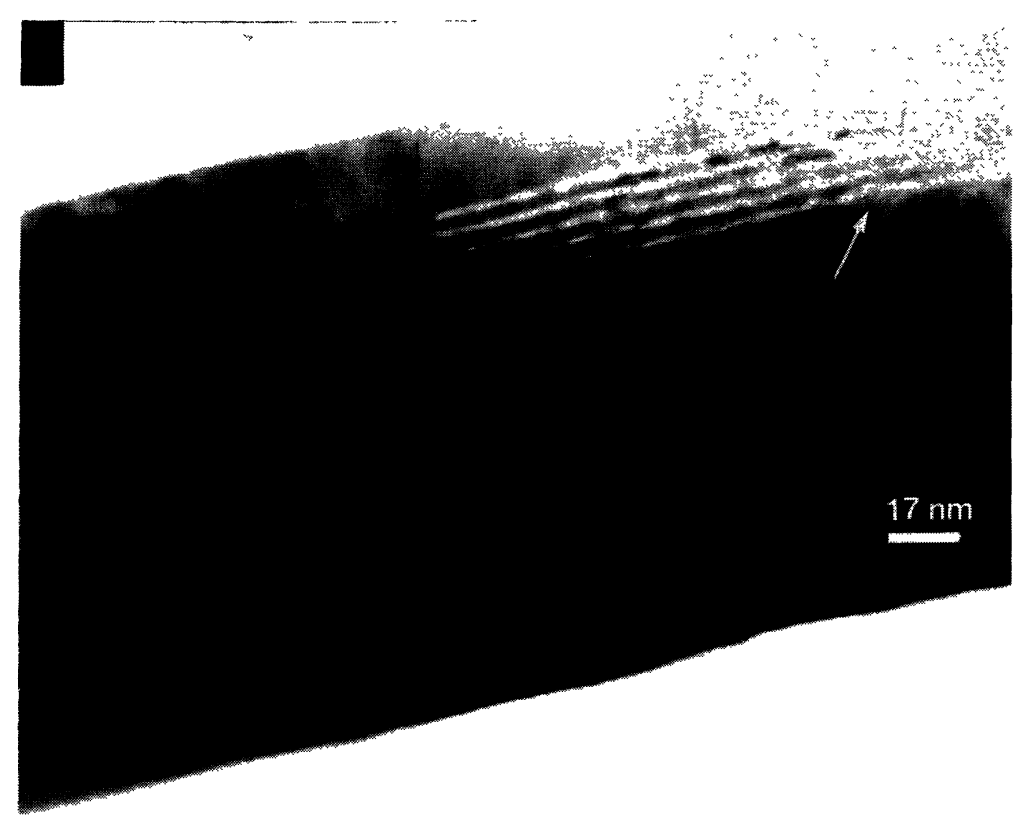

a)

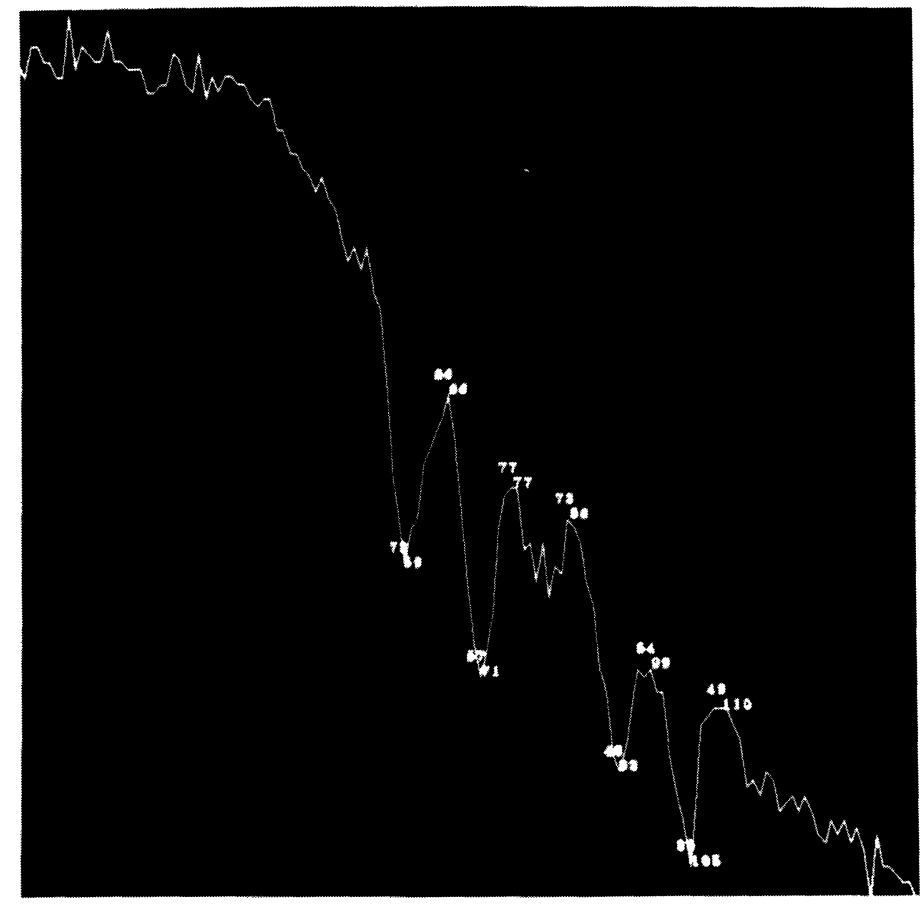

b)

Fig. 1. - a) Cross section bright field image of a pentalayer: the five cobalt layers appear brighter than the gold layers. b) Intensity profile drawn across the pentalayer. 


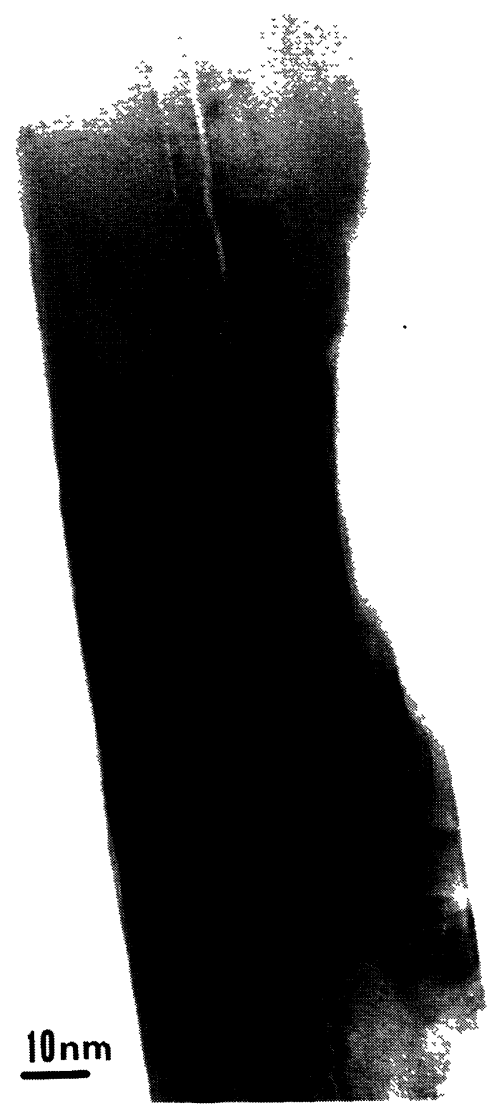

a)

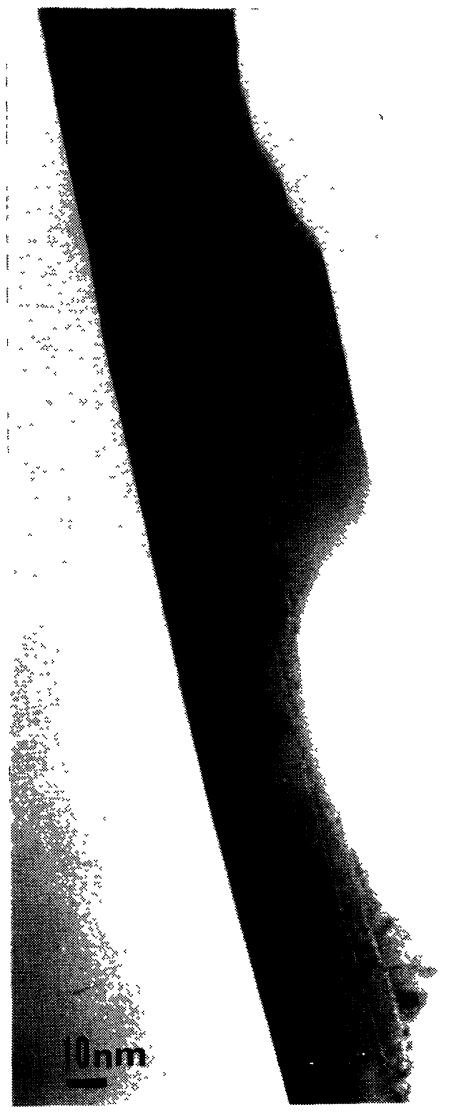

b)

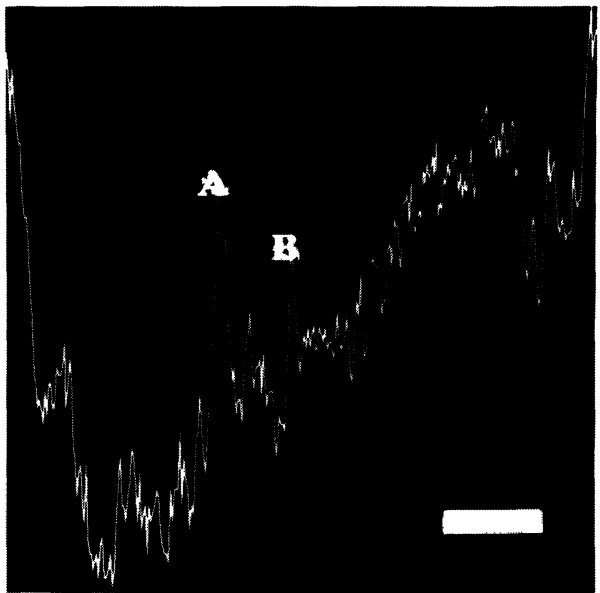

d)

Fig. $2 .-3$ cross views of differente regions of a bilayer: the 2 cobalt films have thicknesses of 0.5 and $1 \mathrm{~nm}$. The arrow shows a grain boundary. d) Intensity profile drawn across the bilayer. 
in figures $2 \mathrm{a}, \mathrm{b}$ and c. With these three micrographs, we can see nearly $500 \mathrm{~nm}$ of the bilayer. All the layers are continuous over long distances, including the first cobalt layer in spite of its thinness, contrary to some other results [4]. In fact, the first cobalt film is rather regular with a weak roughness though the thickness is slightly irregular. This roughness is the same as that of the gold buffer. The second cobalt layer, which is thicker, exhibits some modulations, but these defects seem to arise from the gold spacer and they are not increased by the cobalt film. Both layers are continuous along all the visible regions. A grain boundary is present in micrograph $2 \mathrm{c}$ (see the arrow). It gives rise to some perturbations, such as a shift, mostly in the second layer. This is rather unusual and is the only example we have seen in this sample. Figure $2 \mathrm{~d}$ shows an intensity profile drawn across the bilayer. One can seen that indeed one of the layer has a thickness half the layer thickness.

\section{Discussion and conclusion.}

We have been able to observe very thin cobalt layers in multilayers, over long distances and with a resolution of several angströms: these observations show that the cobalt films, though composed of a small number of atomic planes, are continuous and deposited rather regularly on the first annealed gold layer. Magnetic and resistivity measurements had indeed shown that the layers could be considered as continuous as an average, but these measurements cannot exclude the existence of holes in the films, even large ones. The roughness of the gold substrate or of the other layers being at least of one or two atomic planes, it seemed probable that a $0.5 \mathrm{~nm}$ thick cobalt film (i.e. 2-3 cobalt planes) would show some structural discontinuities. But, even for this very thin film, observed over long distances $(500 \mathrm{~nm})$, we can say that the cobalt thicknesses are rather constant, no holes have been observed and the roughness for both interfaces are the same.

\section{References}

[1] Césari C., Le Dang K., Renard D., Faure J.P., Veillet P. and Nihoul G., J. Magn. Magn. Mater. 78 (1989) 296.

[2] MARLIËRE C., RENARD D and ChauvineaU, Thin Sol. Films 200 (1991).

[3] RENARD D. and NiHOUl G., Philos. Mag. 55 (1987) 75.

[4] HAKKens F., COENE W. and DEN BROEDER F.J.A., MRS spring metting, Anaheim (1991). 